\title{
Comparative Study between Slow Conventional Freezing and Cryoprotectant-Free Vitrification of Human Spermatozoa in Large Volume \\ Mohammed M. Farid Rayea ${ }^{1}$, Medhat Kamel Amer ${ }^{1}$, Ahmed Abdelhady Mousa ${ }^{2}$, Mohammed Gamal Farghaly ${ }^{1 *}$ \\ ${ }^{1}$ Department of Andrology, Sexology and STDs, Faculty of Medicine, Cairo University, ${ }^{2}$ Department of Andrology, Sexology and STDs, Faculty of Medicine, Bani Suef University *Corresponding author: Mohammed Gamal Farghaly, Mobile: (+20) 1003656609,
}

Email: drmohamedgama193@yahoo.co

\section{ABSTRACT}

Background: Cryopreservation is the collection, freezing, and long term storage of sperm, and is a highly effective method of protecting male fertility. Cryopreservation of semen has been widely used as a vital method for fertility preservation of male patients before undergoing chemotherapy, radiotherapy, and/or surgery that may lead to testicular failure or ejaculatory dysfunction.

Objective: The aim of this study was to compare the aseptic technology of cryoprotectant-free vitrification of human spermatozoa in large volume, to conventional freezing protocol as regards post thawing motility, vitality and sperm DNA fragmentation.

Patients and methods: This study included a total of 20 male patients seeking seminal fluid analysis, attending at the andrology laboratory of a specialized IVF center (ADAM International Hospital for Fertility and Sterility, Giza, Egypt. Patients were presented with the diagnoses of normozoospermia, oligozoospermia (either isolated or combined with asthenozoospermia or teratozoospermia).

Results: Motility of (in a large volume $(300 \mu \mathrm{l})$ in the absence of permeable cryoprotectants displayed significant statistically lower levels as compared to conventional Sperm Freezing. It was shown in different groups at different times (post thawing and 1-hour and 24-hour) of assessment that motility of vitrified spermatozoa decreases in comparison with slow conventional freezing as we go from the baseline. DNA fragmentation of vitrified spermatozoa showed higher levels as compared to conventional slow freezing but there is no significant statistical difference between vitrification and conventional slow freezing in DNA fragmentation.

Conclusion: It could be concluded that vitrification technique was quite far away from comparison with slow conventional freezing protocol, and still need for further modifications and wide scale of study to achieve the good results.

Keywords: Slow Conventional Freezing, Cryoprotectant-Free Vitrification, Human Spermatozoa in Large Volume.

\section{INTRODUCTION}

Many advances in reproductive medicine in the past five decades have made cryopreservation of human spermatozoa an invaluable tool for the clinical management of infertility and sperm banking. The advent of in vitro fertilization (IVF) and intracytoplasmic sperm injection (ICSI) with microsurgical sperm handling techniques along with advances in female gamete acquisition have resulted in an increased demand for the cryopreservation of semen and tissue samples, often containing a very limited number of spermatozoa. Sperm cryopreservation also makes it possible for cancer patients to preserve their fertility prior to gonadotoxic chemotherapy or radiation. Applications of sperm banking are not limited to cancer patients but extend to patients undergoing certain types of pelvic or testicular surgeries; those who suffer from degenerative illnesses such as diabetes or multiple sclerosis; spinal cord disease or injury; and persons in occupations where a significant risk of gonadotoxicity prevails ${ }^{(\mathbf{1})}$.

A conventional slow freezing protocol has been in use for many years and very little has changed in terms of methodology and reagents. While freezing aims to preserve cells it can also easily destroy them if certain precautionary steps are not taken into consideration.
During cryopreservation cells and tissue undergo dramatic transformation in chemical and physical characteristics as the temperature drops from +37 to $196^{\circ} \mathrm{C}$. The cells can lose up to $95 \%$ of their intracellular water. The concentration of solutes increases considerably, triggering the possibility of osmotic shock. Moreover, potential intracellular ice crystallization and mechanical deformation by extracellular ice may cause significant injury leading to cell death. Furthermore, if cells survive freezing, they might sustain additional damage during the thawing process due to osmotic shock, uncontrollable swelling and ice re-crystallization ${ }^{(2)}$.

Recently scientists $(3,4,5)$ have begun to reinvestigate the utility of ultra- rapid freezing in the search for alternative methods of sperm cryopreservation. Slow freezing of sperm utilizes cooling rates of $1-10^{\circ} \mathrm{C} / \mathrm{min}$, while the rapid freezing, or vitrification, technique allows for cooling rates to reach more than $40-1000^{\circ} \mathrm{C} / \mathrm{min}$ in order to avoid intracellular ice formation. As new techniques are perfected, there is a potential for sperm cryopreservation to greatly improve in the future ${ }^{(3,4,5)}$.

The aim of this study was to compare the aseptic technology of cryoprotectant-free vitrification of human spermatozoa in large volume, to conventional freezing 
protocol as regards post thawing motility, vitality and sperm DNA fragmentation.

\section{PATIENTS AND METHODS}

This study included a total of 20 male patients seeking seminal fluid analysis, attending at the andrology laboratory of a specialized IVF center (ADAM International Hospital for Fertility and Sterility, Giza, Egypt. Written informed consent of all the subjects was obtained. This study was conducted between January 2017, and January 2018.

\section{Ethical approval:}

The study was approved by the Ethics Board of Aswan University

Patients were presented with the diagnoses of normozoospermia, oligozoospermia (either isolated or combined with asthenozoospermia or teratozoospermia).

Inclusion criteria: Semen analysis showing at least 1 motile sperm/drop after centrifugation, whatever the sperm count and morphology.

Exclusion criteria: Patients with immotile spermatozoa or pyospermia.

Semen samples were collected, and then semen analysis was done according to the WHO ${ }^{(6)}$ manual of semen processing and analysis (5th edition).

The included subjects were divided into two groups; Group A consisted of 10 patients with the diagnosis of normozoospermia, mild or moderate oligozoospermia (Spermatozoa count more than $5 \times 10^{6} / \mathrm{ml}$ ) and prepared with swim up technique., Group B consisted of 10 patients with the diagnosis of severe oligozoospermia either isolated or combined with asthenozoospermia or teratozoospermia (count of or less than $5 \times 10^{6} / \mathbf{m l}$ ) and prepared with wash and centrifuge technique.

Then after proper mixing, each sample is going to be splitted into 3 aliquots as follows:

Aliquot 1: control group (fresh ejaculate)

Aliquot 2: spermatozoa cryopreserved by slow conventional freezing with glycerol-containing medium, Aliquot 3: spermatozoa vitrified in $0.3 \mathrm{~mL}$ insemination "French" straws in culture medium with $0.25 \mathrm{M}$ sucrose. Thawing will be done, and motility, vitality and DNA integrity will be assessed and compared in group A and B.

\section{Sample Preparation:}

Samples were prepared (either by swim up or wash and centrifugation) before further assessment and cryopreservation by wash and centrifugation or by swim up, according to seminal fluid assessment;

Normal, mild and moderate male factor sperms were prepared by swim up while Severe oligozoospermia and severe male factor were prepared by wash and centrifugation ${ }^{(6)}$, (Modified Human tubal fluid $+1 \%$ Human Serum Albumin (mHTF - HAS), pure sperm wash $^{\circledR}$ Nidacon, Sweden) Quinn et al. ${ }^{(7)}$ were the basic medium used in sperm preparation.
The diluted suspension of spermatozoa was again diluted (1:1) with $0.5 \mathrm{M}$ sucrose solution $(0.25 \mathrm{M}$ end concentration). The $0.5 \mathrm{M}$ sucrose in bi-distillate water then filtered with $0.22 \mathrm{~mm}$ filter then was frozen until use. After dilution, aliquots were maintained at $37.8^{\circ} \mathrm{C}$ for $5 \mathrm{~min}$ before the cooling procedure takes place. Then after proper mixing, each sample was split into 3 aliquots: Aliquot 1: control group (fresh ejaculate), Aliquot 2: spermatozoa cryopreserved by slow conventional freezing with glycerol-containing medium, Aliquot 3: spermatozoa vitrified in $0.3 \mathrm{ml}$ insemination "French" straws in culture with $0.25 \mathrm{M}$ sucrose containing medium.

\section{Spermatozoa Cryopreservation:}

1. Spermatozoa Vitrification Technology of aseptic cryoprotectant-free vitrification of human spermatozoa in large volumes; the technology includes - Cryoprotective medium with only nonpermeable cryoprotective agents $(0.25 \mathrm{M}$ sucrose in end concentration). As basal medium was (Modified Human tubal fluid medium $+1 \%$ Human Serum Albumin, Puresperm wash ${ }^{\circledR}$ Nidacon, Sweden). Using of $0.3 \mathrm{ml}$ plastic straws with subsequent sealing from both side before cooling in liquid nitrogen. The warming up of spermatozoa is achieved by immersing straw with vitrified spermatozoa into warmed water bath at $42^{\circ} \mathrm{C}$.

The packaging of spermatozoa for aseptic vitrification was performed in the following way: Spermatozoa suspensions were cooled in $0.3 \mathrm{ml}$ plastic (CBS) straws (CryoBio System, Paris, France). The straw was labeled with asterisk $(1 \mathrm{~cm}$ from the inner end of cotton-polyvinyl plunge, the straw was filled up to asterisk with $0.3 \mathrm{ml}$ of spermatozoa suspension by aspiration. Then the filled straw was expelled from the tube while aspiration of air continued. Subsequently, when the suspension reached the polyvinyl plunge, the polymerization of polyvinyl initiated due to humidification. After aspiration was completed, and the top end of straw was sealed by polymerized polyvinyl, straw was heat-sealed at both sides by thermo-hermetic sealing. The straws were immersed into liquid nitrogen in horizontal position (approximately for 8 seconds) and were stored there at least for 24 hours before use. The warming up of spermatozoa was achieved by immersing straw with vitrified spermatozoa into water bath at $42^{\circ} \mathrm{C}$ and dangling it gently in water for 20 seconds. After warming, the residual fluid was removed from the straw with paper towel, and straw disinfected with $70 \%$ ethanol. The heat-sealed part of straw (opposite to the cotton polyvinyl plunge) was cut off with sterile scissors, and the aspirator was connected with the straw. A low differential negative pressure was applied by aspiration. That ensures that after subsequent cutting of the cotton-polyvinyl plunge fluid won't be leaking out. Finally, the suspension was expelled from the straw for immediate evaluation of sperm quality.

\section{Conventional Spermatozoa Freezing:}

The manual method of conventional freezing was performed by decreasing the temperature of the semen 
after adding a Glycerol cryoprotectant (Sperm Freeze Solution, Fertipro) ( kept at $4-6^{\circ} \mathrm{C}$ and placed at $37^{\circ} \mathrm{C}$ for $10-15 \mathrm{~min}$ before being ready to use), the cryoprotectants was added in an equal volume to semen in a dropwise manner, gently mixed at room temperature, in a stepwise manner and after that loading the specimen in $0.3 \mathrm{ml} \mathrm{CBS}$ straws (CryoBio System, Paris, France) and slowly cooling the specimen then plunging the samples into liquid nitrogen. The initial cooling rate of the specimen from room temperature to $5^{\circ} \mathrm{C}$ was $0.5-1^{\circ} \mathrm{C} / \mathrm{min}$. The sample was then frozen from $5^{\circ} \mathrm{C}$ to $-80^{\circ} \mathrm{C}$ at a rate of $1-10^{\circ} \mathrm{C} / \mathrm{min}$. The specimen was then plunged into liquid nitrogen at $-196^{\circ} \mathrm{C}$. To thaw the samples, the straws were taken from the liquid nitrogen, held in air for 30 seconds, immersed into $37^{\circ} \mathrm{C}$ water bath in the horizontal position and held in this bath for 20 seconds until the ice melted. After thawing, $10 \mathrm{~mL}$ of basic m HTF-HAS medium was added to the thawedsample and centrifuged for 5 minutes at $340 \mathrm{~g}$. The supernatant was removed and the pellet resuspendedwith the same basic medium in order to obtain a final concentration of $0.5 \times 10^{6} / \mathrm{ml}$.

\section{Sperm Assessment:}

Motility of spermatozoa was assessed as soon as the samples liquefied, then post thawing motility was assessed as soon as possible, then after 1 hour and finally. after 24 hours for evaluation of long-term spermatozoasurvival. The hemocytometer was used for most of themotility studies. Motility was estimated under lightmicroscope under x400 magnification.
Vitality "Hypo-osmotic swelling test for human sperm (HOS)"Hypo-osmotic swelling test medium was prepared according to instructions of $\mathbf{W H O}{ }^{(6)}$, and was used for hypo-osmotic swelling test

Vitality of spermatozoa was assessed using the (HOS) test as soon as the samples liquefied, then post thawing vitality was assessed, then after 1 hour and finally after 24 hours for evaluation of long-term spermatozoa survival.

\section{Statistical analysis}

Recorded data were analyzed using the statistical package for social sciences, version 20.0 (SPSS Inc., Chicago, Illinois, USA). Quantitative data were expressed as mean \pm standard deviation (SD). Qualitative data were expressed as frequency and percentage.

\section{The following tests were done:}

Independent-samples t-test of significance was used when comparing between two means.

Chi-square $\left(\mathrm{x}^{2}\right)$ test of significance was used in order to compare proportions between two qualitative parameters.

- The confidence interval was set to $95 \%$ and the margin of error accepted was set to $5 \%$. The p-value was considered significant as the following:

Probability (P-value)

$\mathrm{P}$-value $<0.05$ was considered significant.

$\mathrm{P}$-value $<0.001$ was considered as highly significant.

$\mathrm{P}$-value $>0.05$ was considered insignificant.

\section{RESULTS}

I. Comparison between aliquots regarding motility \%in group A (No=10)

Table (1): Comparison between aliquots regarding motility \% in group $\mathrm{A}$

\begin{tabular}{|c|c|c|}
\hline Time & Aliquot & Mean \pm SD \\
\hline & Prefreezing (Neat semen) & $59.8 \pm 19.9$ \\
\hline \multirow{3}{*}{ 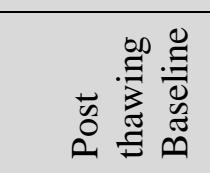 } & Thawing after slow freezing & $17.3 \pm 13.1$ \\
\hline & $\mathrm{VF}($ post thawing) & $3.8 \pm 2.5$ \\
\hline & VF / slow Frozen & $-13.5 \pm 10.6(\mathrm{P}$-value $0,003 *)$ \\
\hline \multirow{3}{*}{ 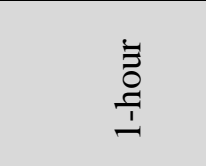 } & Thawing after slow freezing & $13.0 \pm 10.0$ \\
\hline & $\mathrm{VF}$ (post thawing) & $3.1 \pm 3.8$ \\
\hline & VF / slow Frozen & $-9.9 \pm 8.5\left(\mathrm{P}\right.$-value $\left.0,005^{*}\right)$ \\
\hline \multirow{3}{*}{$\stackrel{+}{\Delta}$} & Thawing after slow freezing & $5.7 \pm 7.2$ \\
\hline & $\mathrm{VF}$ (post thawing) & $0.4 \pm 0.5$ \\
\hline & VF / slow Frozen & $-5.3 \pm 6.9\left(p-\right.$ value $\left.0.038^{*}\right)$ \\
\hline
\end{tabular}

\#Paired -test, *Significant

Table 1 show that Motility \% at different times (post thawing and 1-hour and 24- hours) among group A is significantly decreased among vitrification aliquots, followed by slow frozen aliquots compared to Prefreezing(neat semen) aliquots, $\mathrm{P}<0.05$. The above table showing that the motility $\%$ of group A at different times (post thawing and 1-hour and 24- hours) of vitrified spermatozoa decreases statistically with significance in comparison with slow frozen as we go from the baseline(post thawing) where (Slow freezing (17.3 $\pm 13.1 \%$ ), VF $(3.8 \pm 2.5 \%), \mathrm{p}<0.05)$; in fresh Pre-freezing(neat semen)59.8 $\pm 19.9 \%)$. after 1 hour is also statistically different from frozen spermatozoa where (Slow frozen, $13.0 \pm 10.0 \%, \mathrm{VF}, 3.1 \pm 3.8 \%, \mathrm{p}<0.05$ ) And motility rates of vitrified spermatozoa after 24 hours are also statistically different from slow freezing spermatozoa where (slow freezing $5.7 \pm 7.2 \%$, VF, $0.4 \pm 0.5 \%$. p <0.05). 


\section{Comparison between aliquots regarding vitality \% in group $\mathrm{A}(\mathrm{No}=10)$}

Table (2): Comparison between aliquots regarding vitality \% in group $\mathrm{A}$

\begin{tabular}{|c|c|c|}
\hline Time & Aliquot & Mean \pm SD \\
\hline & Pre-freezing(neat semen) & $77.4 \pm 14.0$ \\
\hline \multirow{3}{*}{ 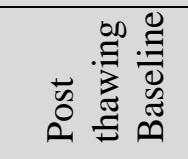 } & Thawing after slow freezing & $30.1 \pm 15.2$ \\
\hline & VF (post thawing) & $14.5 \pm 19.2$ \\
\hline & VF / slow Frozen & $-15.6 \pm 12.2(\mathrm{p}$ value $0.003 *)$ \\
\hline \multirow{3}{*}{$\stackrel{\Xi}{\stackrel{\Xi}{0}}$} & Thawing after slow freezing & $24.9 \pm 14.6$ \\
\hline & VF(post thawing) & $12.1 \pm 19.5$ \\
\hline & VF / slow Frozen & $-12.8 \pm 12.5\left(\mathrm{P}\right.$-value $\left.0.010^{*}\right)$ \\
\hline \multirow{3}{*}{ 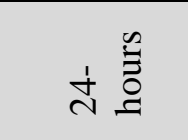 } & Thawing after slow freezing & $13.5 \pm 9.4$ \\
\hline & VF(post thawing) & $12.1 \pm 19.5$ \\
\hline & VF /slow Frozen & $-1.4 \pm 14.6 \quad(p$-value 0.769$)$ \\
\hline
\end{tabular}

\#Paired -test, *Significant

Table 2 show that vitality $\%$ at different times (post thawing(baseline) and 1-hour and 24-hours ) among group A is highest among Pre-freezing aliquots, followed by slow frozen aliquots and least among vitrification aliquots, with statistical significant difference between different aliquots across all times(post thawing(baseline) and 1-hour and 24-hours. The above table show that the mean vitality \% among group A of vitrified spermatozoa decreases significantly in comparison with slow frozen as we go through time, where at the baseline(post thawing) it was (slow frozen
$30.1 \pm 15.2 \%$, VF $14.5 \pm 19.2 \%$. $\mathrm{p}<0.05$; in fresh Prefreezing $77.4 \pm 14.0 \%$ ),

Vitality of vitrified spermatozoa after 1 hour of the same group is also statistically lower than slow frozen

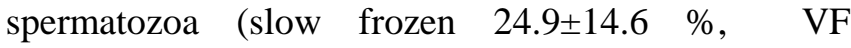
$12.1 \pm 19.5 \%, \mathrm{p}<0.05$; in Pre-freezing $70.3 \pm 14.2$ )

Whereas the vitality of vitrified spermatozoa after 24 hours of the same group is statistically insignificant from slow frozen spermatozoa (slow frozen $13.5 \pm 9.4 \%, \mathrm{VF} 12.1 \pm 19.5 \% \mathrm{p}>0.05$; in Pre-freezing $32.9 \pm 21.3 \%)$.

\section{Comparison between aliquots regarding motility \% in group B $(\mathrm{No}=10)$}

Table (3): Comparison between aliquots regarding motility \% in group B

\begin{tabular}{|c|c|c|}
\hline Time & Aliquot & Mean \pm SD \\
\hline & Pre-freezing(neat semen) & $15.2 \pm 12.3$ \\
\hline \multirow{3}{*}{ 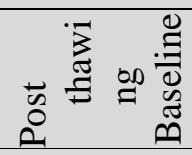 } & Thawing after slow freezing & $4.0 \pm 4.3$ \\
\hline & VF(post thawing) & $0.32 \pm 0.66$ \\
\hline & VF / slow Frozen & $-3.7 \pm 4.1\left(\mathrm{p}\right.$-value $\left.0,019^{*}\right)$ \\
\hline \multirow{3}{*}{\begin{tabular}{l}
$\vdots$ \\
0 \\
\multirow{1}{1}{}
\end{tabular}} & Thawing after slow freezing & $3.0 \pm 3.5$ \\
\hline & VF(post thawing) & $0.22 \pm 0.61$ \\
\hline & VF / slow Frozen & $-2.7 \pm 3.3\left(p\right.$-value $\left.0,029^{*}\right)$ \\
\hline \multirow{3}{*}{ 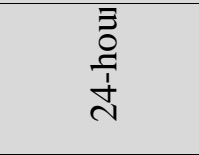 } & Thawing after slow freezing & $0.3 \pm 0.7$ \\
\hline & VF(post thawing) & $0.0 \pm 0.0$ \\
\hline & VF / slow Frozen & $-0.3 \pm 0.7(p$-value 0.165$)$ \\
\hline
\end{tabular}

\#Paired -test, *Significant

Table 3 and figure 3 reveal that the motility\% at different times (post thawing(baseline) and 1-hour and 24hours )among group B was significantly highest among Pre-freezing aliquots, followed by slow frozen aliquots and least among vitrification aliquots, $\mathrm{P}<0.05$.

The same table and figure reveal that the motility of vitrified spermatozoa (in a large volume $(300 \mu \mathrm{l})$ displayed statistically lower levels as compared to conventional slow frozen in all times (post thawing (baseline) and 1-hour and 24-hours ), $\mathrm{P}<0.05$ except at 24 Hours between vitrification versus slow frozen aliquots.

Finally the above table demonstrate that the motility $\%$ of vitrified spermatozoa at the baseline is statistically at lower levels as compared to slow conventional freezing where (slow frozen $4.0 \pm 4.3 \%$, VF $0.32 \pm 0.66 \%$., p <0.05; in fresh Pre-freezing(neat semen) $15.2 \pm 12.3 \%$ ).

Motility of vitrified spermatozoa after 1 hour is statistically different from slow frozen spermatozoa where (slow frozen3.0 $\pm 3.5 \%$, VF0.22 $\pm 0.61 \%$., p $<0.05$; in Pre-freezing(neat semen) $11.9 \pm 11.2 \%$ ). Where as the motility rates of vitrified spermatozoa after 24 hours are statistically insignificant different from slow frozen spermatozoa where (slow frozen $0.3 \pm 0.7 \%$,VF $0.0 \pm 0.0 \%$.p >0.05; in Pre-freezing(neat semen) $6.0 \pm 6.1 \%$ ). 
ejhm.journals.ekb.eg

IV. Comparison between aliquots regarding vitality $\%$ in group $\mathrm{B}(\mathrm{No}=10)$

Table (4): Comparison between aliquots regarding vitality \% in group B

\begin{tabular}{|c|c|c|}
\hline Time & Aliquot & Mean \pm SD \\
\hline & Pre-freezing(neat semen) & $35.3 \pm 19.2$ \\
\hline \multirow{3}{*}{ 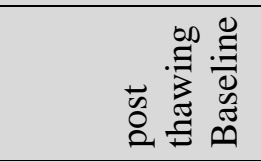 } & Thawing after slow freezing & $17.7 \pm 11.1$ \\
\hline & VF(post thawing) & $3.1 \pm 4.6$ \\
\hline & VF / slow Frozen & $-14.6 \pm 8.8\left(\mathrm{p}-\right.$ value $\left.0.001^{*}\right)$ \\
\hline \multirow{3}{*}{$\frac{1}{\stackrel{3}{0}}$} & Thawing after slow freezing & $13.1 \pm 9.4$ \\
\hline & $\mathrm{VF}($ post thawing $))$ & $2.0 \pm 3.5$ \\
\hline & VF/slow Frozen & $-11.1 \pm 6.8(\mathrm{p}$-value $0.001 *)$ \\
\hline \multirow{3}{*}{ 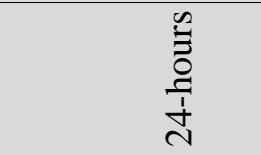 } & Thawing after slow freezing & $7.3 \pm 7.7$ \\
\hline & $\mathrm{VF}$ (post thawing) & $0.6 \pm 1.8$ \\
\hline & VF /slow Frozen & $-6.7 \pm 6.6\left(\mathrm{p}\right.$-value $\left.0.010^{*}\right)$ \\
\hline
\end{tabular}

\#Paired -test, *Significant

Table 4 show that vitality $\%$ at different times(post thawing(baseline) and 1-hour and 24-hour )among group B is highest among Pre-freezing(neat semen)aliquots, followed by slow freezing aliquots and least among vitrification aliquots, with statistical significant difference between different aliquots across all times (post thawing(baseline) and 1-hour and 24-hour). The above table clarify that the mean vitality \% of vitrified spermatozoa at the baseline is statistically lower levels as compared to slow conventional slow freezing where (slow freezing $17.7 \pm 11.1 \%$, VF 3.1 $\pm 4.6 \%$., $\mathrm{p}<0.05$; in fresh Pre-freezing(neat semen) $35.3 \pm 19.2 \%$ ).

Vitality of vitrified spermatozoa after 1 hour is also statistically different from slow freezing spermatozoa (slow

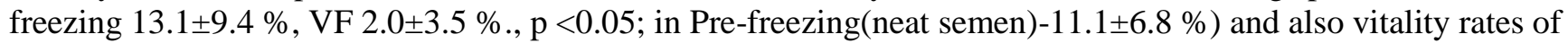
vitrified spermatozoa after 24 hours are statistically different from slow frozen spermatozoa where(slow freezing 7.3 $\pm 7.7 \%$, VF0.6 $\pm 1.8 \%$., $\mathrm{p}<0.05$; in Pre-freezing(neat semen) $22.7 \pm 15.8 \%$ ).

\section{DISCUSSION}

In our study we worked on comparing the aseptic technology of cryoprotectant-free vitrification of human spermatozoa in large volume to conventional slow freezing protocol as regards post-thawing motility and vitality, sperm DNA fragmentation.

Semen samples were collected from male subjects, who were randomly selected from patients seeking seminal fluid analysis at the andrology laboratory at ADAM international fertility hospital, GIZA, Egypt.

Semen analysis were done (WHO 2010), according to results, 20 samples were included in the study they were classified into two groups: (Group 1) 10 samples with the diagnosis of normozoospermia, mild or moderate oligozoospermia. (Spermatozoa count more 5 million $/ \mathrm{ml}$ ). (Group 2) 10 samples with the diagnosis of severe oligozoospermia either isolated or combined with asthenozoospermia or teratozoospermia (count of or less than $5 \mathrm{million} / \mathrm{ml}$ ), or sever male factor cases (count of or less than $1 \mathrm{million} / \mathrm{ml}$ ).

Samples were categorized according to the WHO ${ }^{(6)}$ manual of semen processing and analysis 5 th edition reference values.

Then after proper mixing, each sample was splitted into 3 aliquots as following: Aliquot 1: control group (fresh ejaculate). Aliquot 2: spermatozoa cryopreserved by slow conventional freezing with glycerol-containing medium. Aliquot 3: spermatozoa vitrified in $0.3 \mathrm{~mL}$ insemination "French" straws in culture medium with $0.25 \mathrm{M}$ sucrose

In our study, Motility of vitrified spermatozoa (in a large volume $(300 \mu \mathrm{l})$ in the absence of permeable cryoprotectants displayed significant statistically lower levels as compared to conventional slow freezing. It was shown in different groups at different times (post thawing and 1-hour and 24-hours) of assessment that motility of vitrified spermatozoa decreases in comparison with conventional slow freezing as we go from the baseline. This is contrary to results of The Isachenko's ${ }^{(8)}$ published a report on vitrification of 500 $\mu l$ of human sperm vitrified with $0.25 \mathrm{M}$ sucrose ${ }^{(8)}$.

Katkov et al. ${ }^{(9)}$ found opposite results to Isachenko when vitrified human and bovine spermatozoa in large volume, where they Katkov et al. (9) reported; unfortunately, both human and bovine spermatozoa survived very poorly (single alive spermatozoa were observed) after vitrification in $0.5 \mathrm{ml}$ straws. Interestingly enough, morphology of the sperm was practically intact. Approximately $50 \%$ of human and bull sperm survived slow freezing. The vitrified cells were not visibly damaged, but no motile spermatozoa were observed, for both specie ${ }^{\left({ }^{(9)}\right.}$. In our study, Vitality of vitrified spermatozoa showed significant statistically lower levels as compared to conventional slow freezing .It was shown in different groups that vitality of vitrified spermatozoa decreases significantly in comparison with slow freezing as we go 
through the different times(post thawing and 1-hour and 24-hours).This is contrary to results of another study found that higher rates of CMI were achieved in vitrified sperm as compared to slow conventional freezing. However, as compared to nontreated controls (fresh spermatozoa) both cryopreservation procedures had a significant impact on viability ${ }^{(8)}$.

Some studies showed no statistical differences in parameters such as viability, recovery rate or percentage of morphologically normal spermatozoa with undamaged DNA between vitrified and conventionally frozen cells ${ }^{(\mathbf{1 0})}$.

In our study, DNA fragmentation of vitrified spermatozoa showed higher levels as compared to conventional slow freezing but there is no significant statistical difference between vitrification and conventional slow freezing in DNA fragmentation.

Encouraged by the findings of the German group, the Canadians (as Moskovtsev et al. ${ }^{(1)}$ ) have also looked at possibilities to utilize vitrification in their laboratory. They have compared sperm motility, kinetics and DNA damage between semen samples cryopreserved by standard vapour freezing versus vitrification protocols ${ }^{(\mathbf{1})}$. Their results indicate that sperm motility was significantly reduced for both types of frozen/thawed samples. Mean motility of vitrified samples was decreased when compared to samples prior to freezing, which was almost two-fold higher compared to motility of samples frozen by standard slow vapor protocol, and also was decreased when compared to samples prior to freezing.

Sperm kinematics such as VCL, VSL, and LIN were not significantly different between the two types of cryopreservation protocols without taking into account CASA- paradox.

However, when MKP were calculated, it was revealed that indeed vitrified samples had superior recovery of sperm kinematic parameters in comparison to slow freezing.

They found statistically significant increase in sperm DNA damage after both methods of sperm freezing. However, the increase in DNA damage was minimal and to a degree probably irrelevant to clinical concerns. No significant differences were observed in sperm DNA damage between slow freezing and vitrification. They can confirm from previous reports that human spermatozoa can be successfully vitrified without the use of potentially toxic cryoprotectants. The vitrification protocol showed significantly better results in preserving motility rates of spermatozoa when compared to slow vapour freezing. No significant differences were observed in post thaw sperm DNA damage in comparison to the standard slow freezing method $^{(1)}$.

From the fore-mentioned data we concluded that vitrification technique was quite far away from comparison with old conventional slow freezing protocol, and still need for further modifications and wide scale of study to achieve the recent results reached by the Isachenko.

Our results were concomitant with Katkov's results ${ }^{(9)}$, and we have also some technical differences that we might attribute to our far results from Isachenko'.

The ongoing controversy between scientists and the discrepancy between our results and Isachenko' together with its agreement with Katkov's' will encourage us to continue in research on this new technology.

\section{CONCLUSION}

It could be concluded that vitrification technique was quite far away from comparison with slow conventional freezing protocol, and still need for further modifications and wide scale of study to achieve the good results.

\section{REFERENCES}

1. Moskovtsev SI, Kuznyetsov V, Spiridonov S et al. (2011): Comparison of vitrification and slow vapor protocols for cryopreservation of human spermatozoa. In: Materials of 57th Canadian. Fertility and Andrology Society Annual Meeting. https://cfas.ca > 2011-toronto

2. Sierens J, Hartley JA, Campbell MJ et al. (2002): If In vitro isoflavone supplementation reduces hydrogen peroxide-induced DNA damage in sperm. TeratogCarcinog Mutagen, 22: 227-32.

3. Isachenko V, Isachenko E, Montag M et al. (2005): Clean technique for cryoprotectant - free vitrification of human spermatozoa. Reprod Biomed Online, 10:350-354.

4. Katkov II, Isachenko V, Isachenko E et al. (2006): Lowand high-temperature vitrification as a new approach to biostabilization of reproductive and progenitor cells. International Journal of Refrigeration, 29: 346-357.

5. Merino O, Sanchez R, Risopatron J et al. (2012): Cryoprotectant-free vitrification of fish (Oncorhynchus mykiss) spermatozoa: first report. Andrologia, 44:390-395.

6. WHO (2010):Laboratory manual for the examination and processing of human semen. https://apps.who.int/ iris/handle/10665/44261

7. Quinn P, Warnes GM, Kerin JF et al. (1985): Culture factors affecting the success rate of in vitro fertilization and embryo transfer. Ann NY Acad Sci., 442:195-204.

8. Isachenko V, Maettner R, Petrunkina AM et al. (2011d): Cryoprotectant free Vitrification of Human Spermatozoa in Large (to $0.5 \mathrm{~mL}$ ) Volume: Novel Technology. Clin Lab., 57:643-650.

9. Katkov II, Bolyukh VF, Chernetsov OA et al. (2012): Kinetic Vitrification of Spermatozoa of Vertebrates: What Can We Learn from Nature?, Current Frontiers in Cryobiology, 3:34-8.

10. Punyatanasakchai $\mathbf{P}$, Sophonsritsuk A, Weerakiet $\mathbf{S}$ et al. (2008): Comparison of cryopreserved human sperm in vapor and liquid phases of liquid nitrogen: effect on motility parameters, morphology, and sperm function. Fertil Steril., 90:1978-82. 